\title{
Characterization of foreground emission on degree angular scales for CMB B-mode observations
}

\section{Thermal dust and synchrotron signal from Planck and WMAP data}

\author{
N. Krachmalnicoff ${ }^{1,2}$, C. Baccigalupi ${ }^{3,4}$, J. Aumont ${ }^{5}$, M. Bersanelli ${ }^{1,2}$, and A. Mennella ${ }^{1,2}$ \\ 1 Dipartimento di Fisica, Università degli Studi di Milano, via Celoria, 16, 20133 Milano, Italy \\ e-mail: nicoletta.krachmalnicoff@unimi.it \\ 2 INAF/IASF Milano, via E. Bassini, 15, 20133 Milano, Italy \\ 3 SISSA, Astrophysics Sector, via Bonomea 265, 34136 Trieste, Italy \\ ${ }^{4}$ INFN, via Valerio 2, 34127 Trieste, Italy \\ 5 Institut d'Astrophysique Spatiale, CNRS (UMR 8617) Université Paris-Sud 11, Bâtiment 121, 91405 Orsay, France
}

Received 2 November 2015 / Accepted 28 January 2016

\section{ABSTRACT}

\begin{abstract}
We quantify the contamination from polarized diffuse Galactic synchrotron and thermal dust emissions to the $B$ modes of the cosmic microwave background (CMB) anisotropies on the degree angular scale, using data from the Planck and Wilkinson Microwave Anisotropy Probe (WMAP) satellites. We compute power spectra of foreground polarized emissions in 352 circular sky patches located at Galactic latitude $|b|>20^{\circ}$, each of which covers about $1.5 \%$ of the sky. We make use of the spectral properties derived from Planck and WMAP data to extrapolate, in frequency, the amplitude of synchrotron and thermal dust $B$-mode spectra in the multipole bin centered at $\ell \simeq 80$. In this way we estimate the amplitude and frequency of the foreground minimum for each analyzed region. We detect both dust and synchrotron signal on degree angular scales and at a $3 \sigma$ confidence level in 28 regions. Here the minimum of the foreground emission is found at frequencies between 60 and $100 \mathrm{GHz}$ with an amplitude expressed in terms of the equivalent tensor-to-scalar ratio, $r_{\mathrm{FG}, \min }$, between $\sim 0.06$ and $\sim 1$. Some of these regions are located at high Galactic latitudes in areas close to the ones that are being observed by suborbital experiments. In all the other sky patches where synchrotron or dust $B$ modes are not detectable with the required confidence, we put upper limits on the minimum foreground contamination and find values of $r_{\mathrm{FG}, \mathrm{min}}$ between $\sim 0.05$ and $\sim 1.5$ in the frequency range $60-90 \mathrm{GHz}$. Our results indicate that, with the current sensitivity at low frequency, it is not possible to exclude the presence of synchrotron contamination to CMB cosmological $B$ modes at the level requested to measure a gravitational waves signal with $r \simeq 0.01$ at frequency $\lesssim 100 \mathrm{GHz}$ anywhere. Therefore, more accurate data are essential in order to better characterize the synchrotron polarized component and, eventually, to remove its contamination to CMB signal through foreground cleaning.
\end{abstract}

Key words. cosmic background radiation - cosmology: observations - submillimeter: ISM - polarization - diffuse radiation

\section{Introduction}

The study and characterization of the polarized cosmic microwave background (CMB) signal represents one of the greatest challenges for modern observational cosmology. In particular, the existence of tensor perturbations in the CMB temperature and polarization signal, generated by primordial gravitational waves $(\mathrm{GWs})$, is predicted within the inflationary scenario of the Early Universe, where a transient vacuum energy phase provides accelerated expansion and quantum generation of cosmological perturbations. The amplitude of these perturbations is often parametrized by the tensor-to-scalar ratio $r$. Measurements, or constraints, of this quantity are essential to distinguish among different theories (Planck Collaboration XX 2015).

In recent years, several experiments have been designed and built with the major scientific goal of detecting the effect of GWs into CMB anisotropies, focusing, in particular, on characterizing the curl component of the CMB polarized signal, the $B$-mode (see BICEP2/Keck and Planck Collaborations 2015 , and references therein). The contribution from primordial GWs is relevant on angular scales larger than about one degree. On arcminute scales, the dominant contribution is given by gravitational lensing (GL) owing to deflection of CMB photons by cosmological structures along their travel to the observer. Experiments looking at $\mathrm{CMB} B$ modes include the European Space Agency (ESA) Planck satellite (Planck Collaboration I 2015), ground-based experiments like PolarBear (The Polarbear Collaboration 2014), the Simons Array (Suzuki et al. 2016), BICEP2 (BICEP2 Collaboration I 2014), the Keck Array (BICEP2 and Keck Array Collaborations 2015), SPT (Benson et al. 2014), Advanced ACTPol (Calabrese et al. 2014), QUBIC (QUBIC Collaboration 2011), QUIJOTE (Génova-Santos et al. 2015), CLASS (Essinger-Hileman et al. 2014), and balloonborne instruments, such as EBEX (Chapman et al. 2014), SPIDER (Fraisse et al. 2013), and LSPE (LSPE Collaboration 2012)) $)^{1}$.

These experimental efforts have provided us with upper limits on the value of $r$. The most stringent ones come from Planck temperature and polarization data, yielding $r<0.11$ at 95\% C.L. (Planck Collaboration XIII 2015), and from the BICEP2/Keck/Planck joint analysis of the $B$-mode polarized

1 A complete list of operating and planned CMB experiments is available at http://lambda.gsfc.nasa.gov/product/expt/ 
signal, resulting in $r<0.12$ at $95 \%$ C.L. (BICEP2/Keck and Planck Collaborations 2015). The latter result is particularly relevant because it comes from direct $B$-mode measurements. While this work was completed, a slightly more stringent upper limit was published, which exploits the latest data from the Keck array and BICEP2 telescopes, with $r<0.09$ at 95\% C.L. (BICEP2 and Keck Array Collaborations 2016).

The detection of the CMB cosmological $B$-mode signal, on the angular scales where it should dominate the signature generated by GL, is made difficult not only by the faintness of the signal itself (fraction of $\mu \mathrm{K}$ ), but also by the presence of Galactic polarized foregrounds. Two main kinds of Galactic foreground radiation emit strong linearly polarized signal: the thermal emission of Galactic dust and the synchrotron radiation. For a detailed description of these processes we refer to Planck Collaboration X (2015), Planck Collaboration Int. XXX (2016) and Planck Collaboration XXV (2015).

Thermal radiation from the Galactic interstellar medium dominates the sky signal at frequencies above about $100 \mathrm{GHz}$, and it is partially linearly polarized. The polarization fraction increases with Galactic latitude and can reach the $\sim 20 \%$ level in several large regions of the sky (Planck Collaboration Int. XIX 2015). Its frequency spectrum of thermal dust radiation is described by a gray body, $I_{\mathrm{d}}(v) \propto v^{\beta_{\mathrm{d}}} B_{v}\left(T_{\mathrm{d}}\right)$, where $B_{v}\left(T_{\mathrm{d}}\right)$ is the black body spectrum at temperature $T_{\mathrm{d}}$. In Planck Collaboration Int. XXII (2015), the Planck and WMAP data are used to determine the spectral index $\beta_{\mathrm{d}}$ of the thermal dust emission both in intensity and polarization, finding $\beta_{\mathrm{d}} \simeq 1.59$ for $T_{\mathrm{d}} \simeq 19.6 \mathrm{~K}$.

Synchrotron emission results from the acceleration of cosmic-ray electrons in the Galactic magnetic field. It dominates the sky signal at low frequency ( $\lesssim 100 \mathrm{GHz}$ ). It is strongly linearly polarized, up to $\sim 20 \%$ at intermediate and high Galactic latitudes (Kogut et al. 2007), and shows a power-law frequency dependence with mean spectral index $\beta_{\mathrm{s}} \simeq-3$ (Fuskeland et al. 2014).

The relevance of foreground emission as contaminant for CMB polarization measurements, potentially at all frequencies and all Galactic latitudes, has been claimed in early studies (Baccigalupi 2003) and confirmed, on large sky fractions, by WMAP observations (Page et al. 2007; Gold et al. 2011) and by Planck data (Planck Collaboration X 2015). Recently, Choi \& Page (2015) have used Planck and WMAP data to study the spatial correlation between synchrotron and dust emission and find a positive correlation at $\sim 20 \%$ level on intermediate angular scales $\left(30<\ell<200\right.$ and $\left.f_{\text {sky }}>0.5\right)$. They also find that, considering large portions of the sky, the minimum of foreground emission lies at a frequency around $75 \mathrm{GHz}$ and stress how new data of synchrotron radiation, with higher sensitivity, are needed to completely understand the actual foreground contamination at frequency around $90 \mathrm{GHz}$. The analysis reported in BICEP2/Keck and Planck Collaborations (2015) indicates that most, if not all, of the $B$-mode excess measured by the BICEP2/Keck array data, originally interpreted as GWs signature (BICEP2 Collaboration I 2014), has to be assigned to the Galactic foreground emission. This result confirms, once again, how the characterization of polarized foregrounds represents a crucial aspect for current and future observations of the polarized CMB signal.

In Planck Collaboration Int. XXX (2016, hereafter PIP-XXX), the characterization of the polarized thermal dust radiation at intermediate and high Galactic latitudes is reported, focusing, in particular, on its relevance for CMB polarization measurements. In this work we extend that analysis to also include synchrotron radiation, largely based on the data taken in the $30 \mathrm{GHz}$ band by the Low Frequency Instrument (LFI) onboard Planck, and the WMAP- $K$-band at $22 \mathrm{GHz}$. Our goal is to describe the contamination from foreground emissions on degree angular scales exploiting the current available data. This, in particular, would be useful for optimizing the observation strategies (e.g., frequency channels and observing region) for forthcoming and future suborbitals CMB experiments. To achieve this goal, we analyze thermal dust and synchrotron polarization power spectra with a common methodology on 352 sky regions located at intermediate and high Galactic latitudes. In our analysis we reproduce the PIP-XXX results on thermal dust radiation and combine them with information about synchrotron radiation. In this way we estimate, for each considered sky region, the expected level and range of frequencies where the foreground emission reaches its minimum.

The paper is organized as follows. In Sect. 2 we present the data used for the analysis and we briefly describe the Xpure power spectrum estimator. In Sect. 3 we report our analysis procedure and discuss the results concerning the impact of foreground emission on CMB $B$-mode measurements. Section 4 summarizes the main conclusions.

\section{Data and methodology}

We use the publicly available Planck and WAMP-9 years data to get information on the the Galactic thermal dust radiation and synchrotron emission. The Planck satellite performed full sky observations in linear polarization in seven different frequency bands (centered at the nominal frequencies of 30,44 , $70,100,143,217$, and $353 \mathrm{GHz}$ ). A description of the full set of maps is reported in Planck Collaboration II (2016), Planck Collaboration VI (2016), Planck Collaboration VII (2016), and Planck Collaboration VIII (2016). WMAP observed the sky in five frequency bands, namely $K, K a, Q, V$, and $W$, covering a frequency interval from $\sim 20$ to $\sim 106 \mathrm{GHz}$ (Bennett et al. 2013).

In particular, we consider the Planck-HFI $353 \mathrm{GHz}$ maps as tracers of the Galactic thermal dust emission. Specifically, we used the time-split "Half-Mission" (HM) maps at $353 \mathrm{GHz}$ for estimating power spectra. This pair of maps has highly independent noise, and the cross-correlation between them leads to power spectra with a negligible noise bias. For synchrotron, we considered the Planck-LFI $30 \mathrm{GHz}$ and WMAP- $K$-band maps, which are cross-correlated to estimate power spectra. To check the consistency of results, we also evaluated the synchrotron amplitude by cross-correlating the Planck-LFI $30 \mathrm{GHz} \mathrm{HM}$ maps (year1+year2 and year3+year4 maps) without including WMAP data. To the maps we applied a point source mask that filters out the polarized sources included in the Second Planck Catalogue of Compact Sources (PCCS2) at frequencies of 30 and $353 \mathrm{GHz}$ (Planck Collaboration XXVI 2016).

Table 1 reports the main parameters of these maps, while Fig. 1 shows the polarization amplitude maps $\left(P=\sqrt{Q^{2}+U^{2}}\right.$, with $Q$ and $U$ the two Stokes parameters describing the linear polarization state of incoming radiation), at the latitudes of interest here, corresponding to $|b| \geq 20^{\circ}$. The maps have been smoothed by considering a Gaussian circular beam of $2^{\circ}$, which corresponds to multipole $\ell \lesssim 80$, the angular scale at which the CMB GWs $B$ modes dominate the GL signal.

We characterize the foreground emission on degree angular scales by computing polarization power spectra on a set of smallsized masks, similar to the one used in PIP-XXX: 352 sky regions obtained considering circles with radius $14^{\circ}$ (area of about $600 \mathrm{deg}^{2}$ ) centered on the pixels of the HEALPix $N_{\text {side }}=8$ grid 
Table 1. Characteristics of the WMAP and Planck sky maps used in this analysis (Bennett et al. 2013; Planck Collaboration I 2015).

\begin{tabular}{|c|c|c|c|}
\hline & Planck-353 GHz & Planck-30 GHz & WMAP- $K$-band \\
\hline Central frequency $[\mathrm{GHz}]$ & 353 & 28.4 & 22.4 \\
\hline Beam FWHM [arcmin] & 4.8 & 33.2 & 52.8 \\
\hline Mean Q/U total RMS $\left(2^{\circ},|b|>20^{\circ}\right)^{a} \quad[\mu \mathrm{K}]$ & 49.8 & 6.8 & 16.5 \\
\hline Mean $\mathrm{Q} / \mathrm{U}$ noise RMS $\left(2^{\circ},|b|>20^{\circ}\right)^{a}[\mu \mathrm{K}]$ & 3.3 & 2.0 & 2.2 \\
\hline
\end{tabular}

Notes. ${ }^{(a)}$ The noise level refers to an angular resolution of $2^{\circ}$, assuming a flat $C_{\ell}$ spectrum.
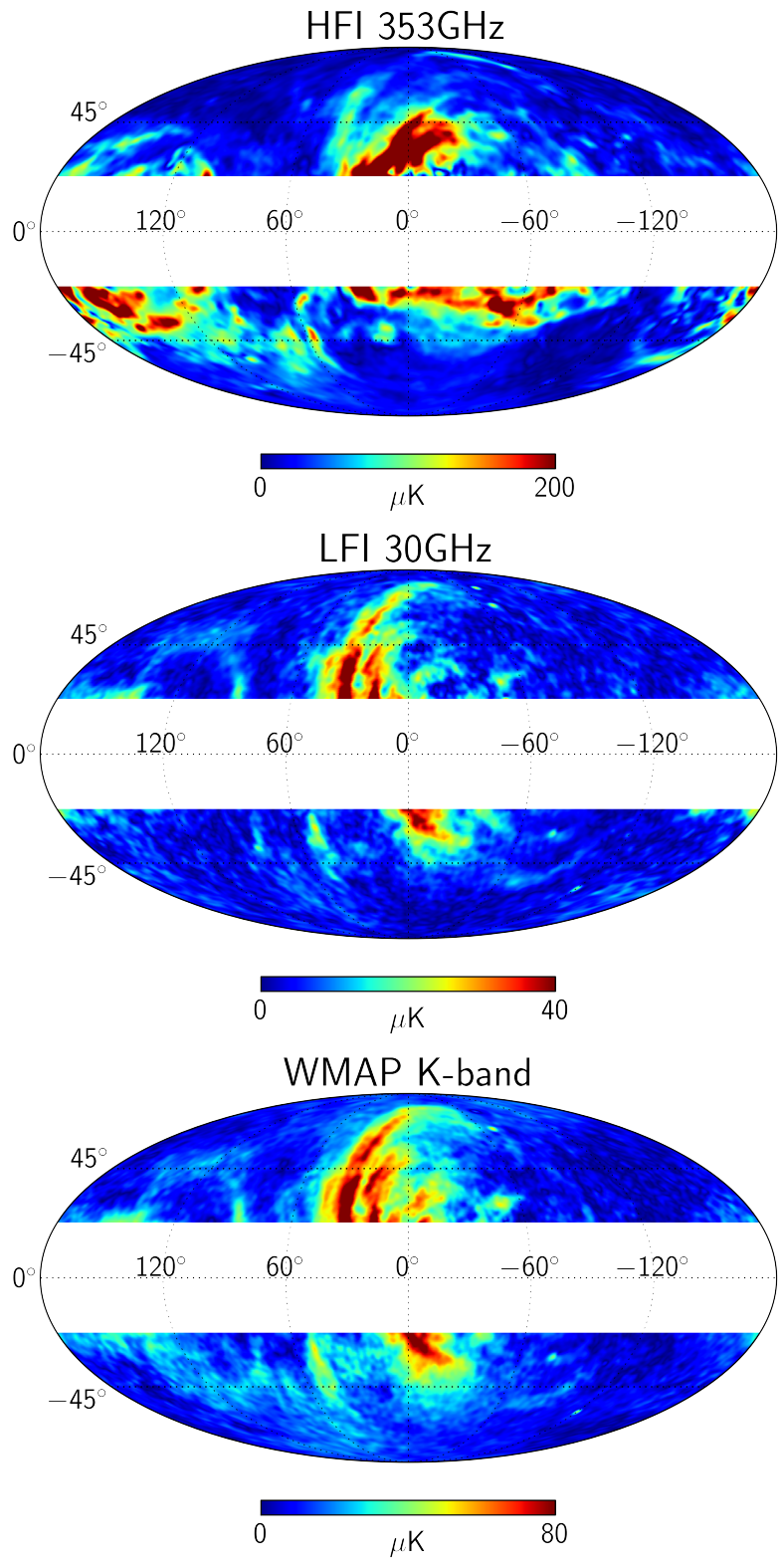

Fig. 1. Amplitude of polarized emission $\left(P=\sqrt{Q^{2}+U^{2}}\right)$ for Planck $353,30 \mathrm{GHz}$ maps (top and middle panels), and WMAP- $K$-band map (bottom panel), in the area considered in this work $\left(|b|>20^{\circ}\right)$. Maps are in thermodynamical CMB units and have been smoothed to $2^{\circ}$ FWHM.

(Górski et al. 2005) with Galactic latitude $|b|>35^{\circ}$. Each of the obtained patches covers about $1.5 \%$ of the sky, a typical dimension for the observation deep fields of ground-based or balloon-borne experiments. The results of the analysis on these sky patches are presented in Sect. 3 .
We also evaluated the amplitude of foreground emissions on larger angular scales by computing polarization power spectra on a different sets of 44 larger sky masks. These masks were obtained considering circular sky regions with radius of $28^{\circ}$ centered on the pixel centers of the HEALPix $N_{\text {side }}=4$ grid and including only those pixels located at Galactic latitude $|b|>50^{\circ}$. This set of masks covers, like the previous one, the portion of the sky at intermediate and high Galactic latitudes with $|b| \gtrsim 20^{\circ}$, and each of the mask has $f_{\text {sky }} \simeq 0.06$.

\subsection{Xpure power spectrum estimator}

The computation of angular power spectra on incomplete sky coverage requires the application of specific algorithms in order to correct for the multipole mixing and the polarization state mixing (E-to-B leakage) derived from the sky cut. A variety of procedures have been proposed and implemented in data analysis algorithms that are commonly used: see, e.g., the Xspect and Xpol codes (Tristram et al. 2005). In this paper, we have adopted the $\mathrm{Xpure}^{2}$ methodology, which is a numerical implementation of the pseudo-pure approach described in Smith (2006) and Grain et al. (2009). It uses a suitable apodization of the considered sky patch that vanishes at the border along with its first derivative. By calculating the spin-weighted windows of the input window function, it estimates the pure- $C_{\ell}$, which ideally are free from the E-to-B leakage. The Xpure code can handle multiple maps for computing auto and cross power spectra.

In our analysis we make use of a cosine squared apodization of the sky masks with an apodization scale equal to $2^{\circ}$ for all the circular sky patches.

\section{Foreground contamination to CMB B-modes}

In this section we describe the results for the estimation of the contamination arising from foreground emission on $\mathrm{CMB}$ $B$-mode observations. To assess this level of contamination, we computed power spectra of the foreground emissions on the set of 352 circular sky masks described previously. Power spectra are computed with Xpure on these regions, specifically in the $\ell$-bins defined by the intervals between multipoles: 20, 60, $100,140,180$, and 220 . Nevertheless, since we are interested in the contamination to $\mathrm{CMB}$ measurements of primordial tensor modes on degree angular scales, we mainly consider the amplitude of foreground emission recovered in the multipole bin centered at $\ell \simeq 80$, hereafter referred to as $\ell_{80}$, where the socalled CMB $B$-mode "recombination bump" peaks and where a number of current and forthcoming suborbital experiments are focused to constrain $r$.

We recall that we estimate thermal dust spectra by crosscorrelating the HFI $353 \mathrm{GHz} \mathrm{HM}$ maps, while for synchrotron

\footnotetext{
2 WWW.apc.univ-paris7.fr/APC_CS/Recherche/Adamis/ MIDASQ9/software/pures2hat/pureS2HAT . html
} 

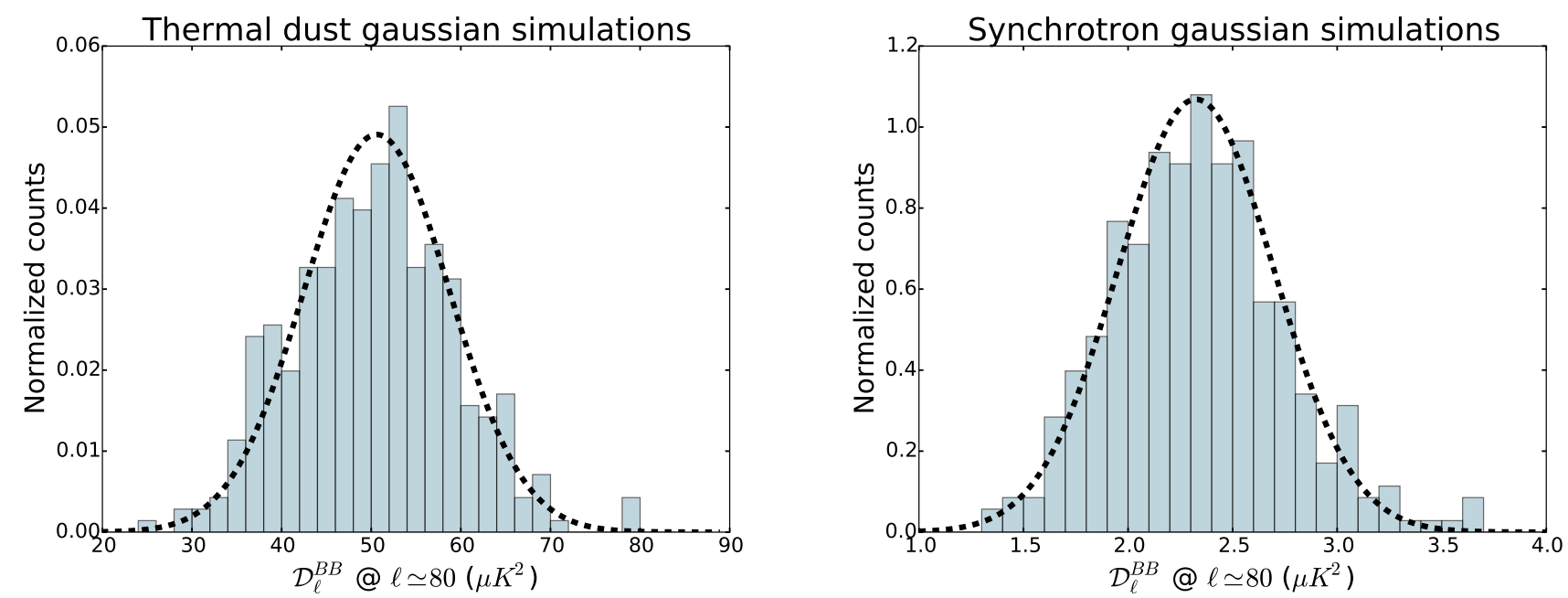

Fig. 2. Validation of the Xpure power spectrum estimator for thermal dust (left panel) and synchrotron (right panel) Gaussian simulations. Gray histograms are obtained from the $B$-mode power spectra estimation of the input simulated maps in the multipole bin centered at $\ell \simeq 80$ in the 352 considered sky regions. Black curves represent the expected Gaussian distribution centered on the mean value of the input spectra from which simulated maps are computed, and with variance defined as in Eq. (1).

we cross-correlate the LFI $30 \mathrm{GHz}$ and WMAP- $K$ maps, therefore evaluating the synchrotron polarization amplitude at the effective frequency $v_{\mathrm{eff}}=\sqrt{v_{\mathrm{LFI}-30} \times v_{\mathrm{WMAP}-\mathrm{K}}} \simeq 25.4 \mathrm{GHz}$.

\subsection{Xpure validation}

We validate the performance of the Xpure algorithm, on our analysis setup, by computing power spectra of simulated polarization maps. We checked whether the spectra amplitude estimation in the $\ell_{80}$ bandpower, calculated in such small sky patches, is unbiased and has minimum variance. Simulated maps are generated considering Gaussian realizations of input theoretical power spectra in the form $\mathcal{D}_{\ell}^{E E / B B}=A^{E E / B B} \ell^{\alpha+2}$ (where $\mathcal{D}_{\ell}^{E E / B B} \equiv$ $\left.\ell(\ell+1) C_{\ell}^{E E / B B} / 2 \pi\right)$. This kind of dependence for polarization foreground power spectra has been found to be valid for both dust emission, with $\alpha \simeq-2.42$ and $\left(A^{B B} / A^{E E}\right) \simeq 0.52$ (Planck Collaboration Int. XXX 2016), and synchrotron radiation, with $\alpha \simeq-2.37$ and $\left(A^{B B} / A^{E E}\right) \simeq 0.36$ (Planck Collaboration X 2015). We therefore explored these two cases in our simulations. Even though the hypothesis of Gaussianity does not apply to the Galactic emissions, these simulations give us the possibility of constructing maps with known expected mean value and variance of the power spectra in a given multipole bin, to be compared with the values retrieved by Xpure.

We calculated power spectra for the two different cases: using maps simulating a Gaussian thermal dust signal having an input power spectrum taking the value $\mathcal{D}_{\ell_{80}}^{B B}=50 \mu \mathrm{K}^{2}$, and maps simulating a Gaussian synchrotron signal with $\mathcal{D}_{\ell_{80}}^{B B}=$ $2.3 \mu \mathrm{K}^{2}$. These numbers represent the median values of the actual $B$-mode amplitude of dust and synchrotron spectra computed from Planck and WMAP data ${ }^{3}$ (see Sect. 3.2). We simulated 352 maps for each case and then computed power spectra on our set of 352 regions, therefore using a different simulated map for each region. Figure 2 shows the results of the validation, with the histogram of the Xpure estimation of the $B$-mode amplitude in the 352 sky regions in the $\ell_{80}$ bin, compared with the Gaussian distribution having a mean equal to the value of the

\footnotetext{
3 Median values are computed considering only those regions where $\mathcal{D}_{\ell_{80}}^{B B}>0$.
}

input spectrum in the same $\ell$ bin and as variance the approximation of the signal sample variance defined as

$$
\operatorname{var}\left(\mathcal{D}_{\ell_{\text {bin }}}^{B B}\right)=\frac{2}{\left(2 \ell_{\text {bin }}+1\right) f_{\text {sky }}^{\text {eff }} \Delta \ell_{\text {bin }}}\left(\mathcal{D}_{\ell_{\text {bin }}}^{B B}\right)^{2},
$$

where $f_{\text {sky }}^{\text {eff }}$ is the mean effective sky fraction retained by the masks, which is approximately ${ }^{4} 0.0125$.

In both cases the histogram recovered from data agrees with the expected Gaussian distribution, with $p$ values obtained from a statistical null-hypothesis test equal to 0.48 and 0.52 for thermal dust and synchrotron cases, respectively ${ }^{5}$ (the analysis of $E$-mode spectra gives similar results). From this validation we can therefore conclude that Xpure estimates the power spectra amplitude correctly in the $\ell_{80}$ bandpower in the considered sky patches.

\subsection{Foreground amplitude in the $\ell_{80}$ bandpower}

Maps in Fig. 3 were constructed from the Xpure evaluation of power spectra at $\ell \simeq 80$ of Planck and WMAP maps. In particular, for both the $\mathrm{HFI}_{353} \mathrm{HM}_{1} \times \mathrm{HM}_{2}$ and $\mathrm{LFI}_{30} \times \mathrm{WMAP}_{K}$ cases, each pixel shows the value of the $E$ or $B$-mode power spectra in the $\ell_{80}$ bandpower, computed in the corresponding circular region with a radius of $14^{\circ}$. The maps show the power spectrum amplitude for those pixel where there is a positive detection of signal at $3 \sigma$ (colored scale on maps). Errors are obtained from one hundred simulations of white noise maps generated from the $Q$ and $U$ pixel-pixel covariance matrices of the input sky maps. In all cases where the power spectrum at $\ell \simeq 80$ does not exceed $3 \sigma$, the maps report upper limits (gray scale on maps).

4 The retained effective sky fraction is not exactly the same for each region, since compact sources are masked, and it varies between 0.0115 and 0.0126 .

$5 \quad p$ value means the probability of getting the observed distribution or a "more unlikely" one if data are indeed coming from the previously defined Gaussian probability function. 

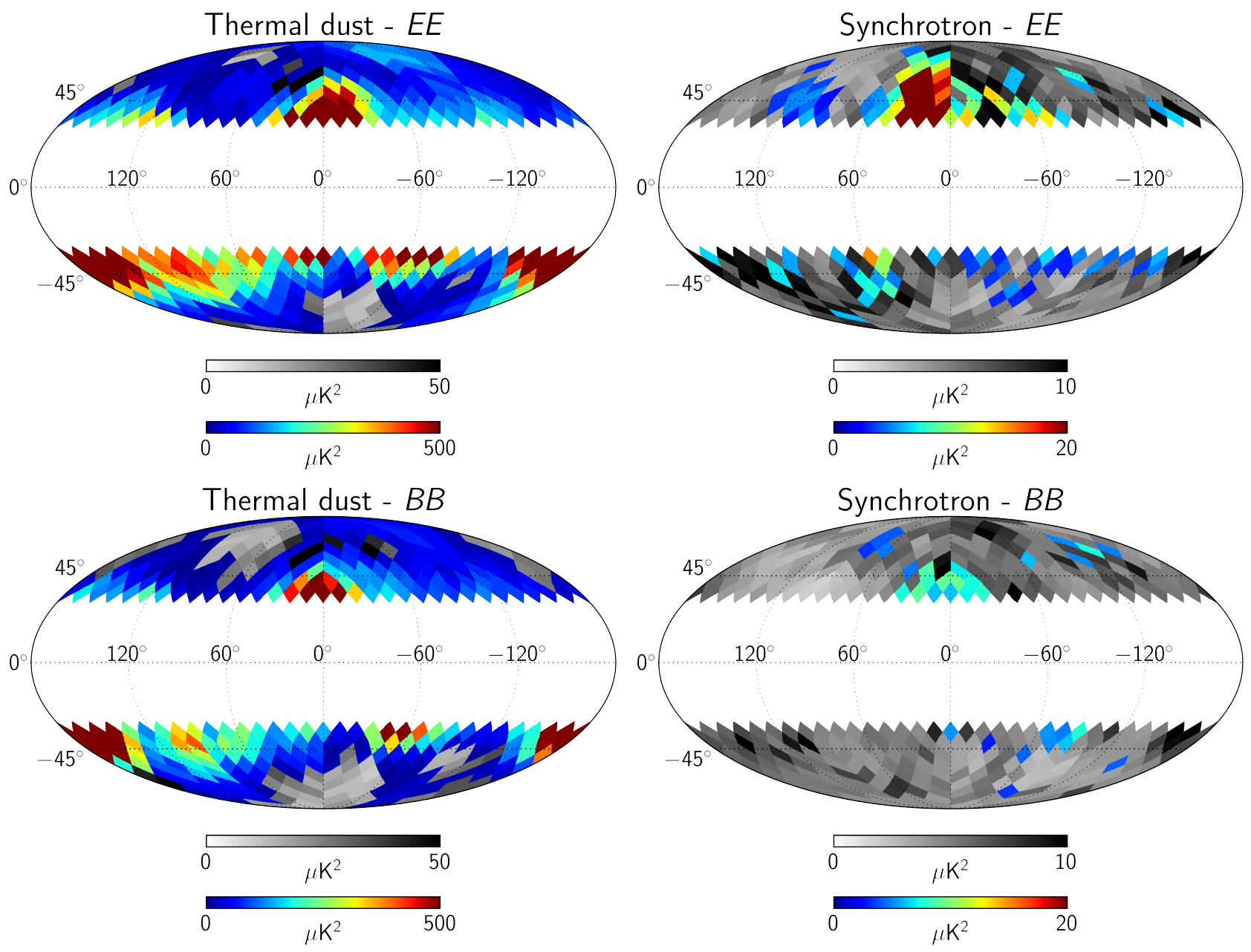

Fig. 3. $E$ (top) and $B$ modes (bottom) power spectra measurements in the $\ell_{80}$ multipole bin for dust in the Planck $353 \mathrm{GHz}$ channel (left) and synchrotron (right) via cross-correlation of the LFI $30 \mathrm{GHz}$ and WMAP- $K$-band. Colored pixels report detection above $3 \sigma$. Gray pixels show upper limits where no detection was possible above the threshold, as defined in Eq. (2).

Upper limits are defined in the following conservative way:

- if $\mathcal{D}_{\ell_{80}}^{B B / E E}>0: \mathrm{UL}\left(\mathcal{D}_{\ell_{80}}^{B B / E E}\right)=\mathcal{D}_{\ell_{80}}^{B B / E E}+3 \sigma\left(\mathcal{D}_{\ell_{80}}^{B B / E E}\right)$

- if $\mathcal{D}_{\ell_{80}}^{B B / E E}<0: \mathrm{UL}\left(\mathcal{D}_{\ell_{80}}^{B B / E E}\right)=3 \sigma\left(\mathcal{D}_{\ell_{80}}^{B B / E E}\right)$.

For both thermal dust and synchrotron the number of positive detections is greater for $E$ than $B$ modes, as expected given the $E / B$ asymmetry of the two kinds of emission (Planck Collaboration Int. XXX 2016; Planck Collaboration X 2015). For thermal dust, positive detections are obtained in 325 and 278 regions for $E$ and $B$ modes, respectively, with values ranging from $\sim 10$ to more than $2500 \mu \mathrm{K}^{2}$ (errors between $\sim 2$ and $\sim 10 \mu \mathrm{K}^{2}$ ). Upper limits range between $\sim 10$ and $\sim 60 \mu \mathrm{K}^{2}$. For $B$ modes, the recovered amplitudes are consistent with the similar ones reported in PIP-XXX, even though the circular regions we consider here are slightly larger with discrepancies above $3 \sigma$ only in ten sky patches.

The situation for synchrotron is more critical, because of the lower signal-to-noise ratio of the data. Positive detections are found only in 101 regions for $E$ modes and 32 regions for $B$ modes, with amplitudes between $\sim 3$ and $\sim 40 \mu \mathrm{K}^{2}$ (errors between $\sim 0.6$ and $\sim 3 \mu \mathrm{K}^{2}$ ). Upper limits range between $\sim 2$ and $\sim 10 \mu \mathrm{K}^{2}$. The number of regions with positive detection for synchrotron $B$ modes increases to 66 or 114 if we consider threshold at $2 \sigma$ or $1 \sigma$, respectively.
The morphology of the retrieved maps follows the morphology of the foreground emissions, with larger amplitude in the $\ell_{80}$ bandpower in those regions where the total polarized signal is stronger (compare with Fig. 1).

\subsection{Minimum of foreground emission}

We now use the results described above to evaluate, for each sky region, the expected contamination arising from foregrounds to CMB $B$ modes measurements. Our goal is to estimate, for each region, the frequency at which the total polarized foreground emission reaches its minimum and its corresponding amplitude. To achieve this goal, we extrapolate the $B B$ maps of Fig. 3 in frequency.

The frequency scaling is computed using the available information on the spectral energy distribution (SED) of thermal dust and synchrotron radiations in polarization. For thermal dust, we adopt a modified blackbody with $\beta_{\mathrm{d}}=1.59 \pm 0.17$ at $T_{\mathrm{d}}=19.6 \mathrm{~K}$ (Planck Collaboration Int. XXII 2015; Planck Collaboration Int. XXX 2016). We rescale the synchrotron radiation considering a power law with spectral index $\beta_{\mathrm{s}}=-3.12 \pm$ 0.04, as reported in Fuskeland et al. (2014) for polarized emission at intermediate and high Galactic latitudes. In extrapolating the foreground contribution at the various frequencies, we take the Planck 353 and $30 \mathrm{GHz}$ and WMAP- $K$-band color 
Table 2. Definition of classes for sky regions.

\begin{tabular}{llcc}
\hline \hline Class & Number of regions & Color scale in Fig. 5 \\
\hline 1. & Detection of thermal dust and synchrotron & 28 & Green \\
2. & Detection of thermal dust, upper limit on synchrotron & 250 & Grey \\
3. & Detection of synchrotron, upper limit on thermal dust & 4 & Blue \\
4. & Upper limit on thermal dust and synchrotron & 70 & Red \\
\hline
\end{tabular}
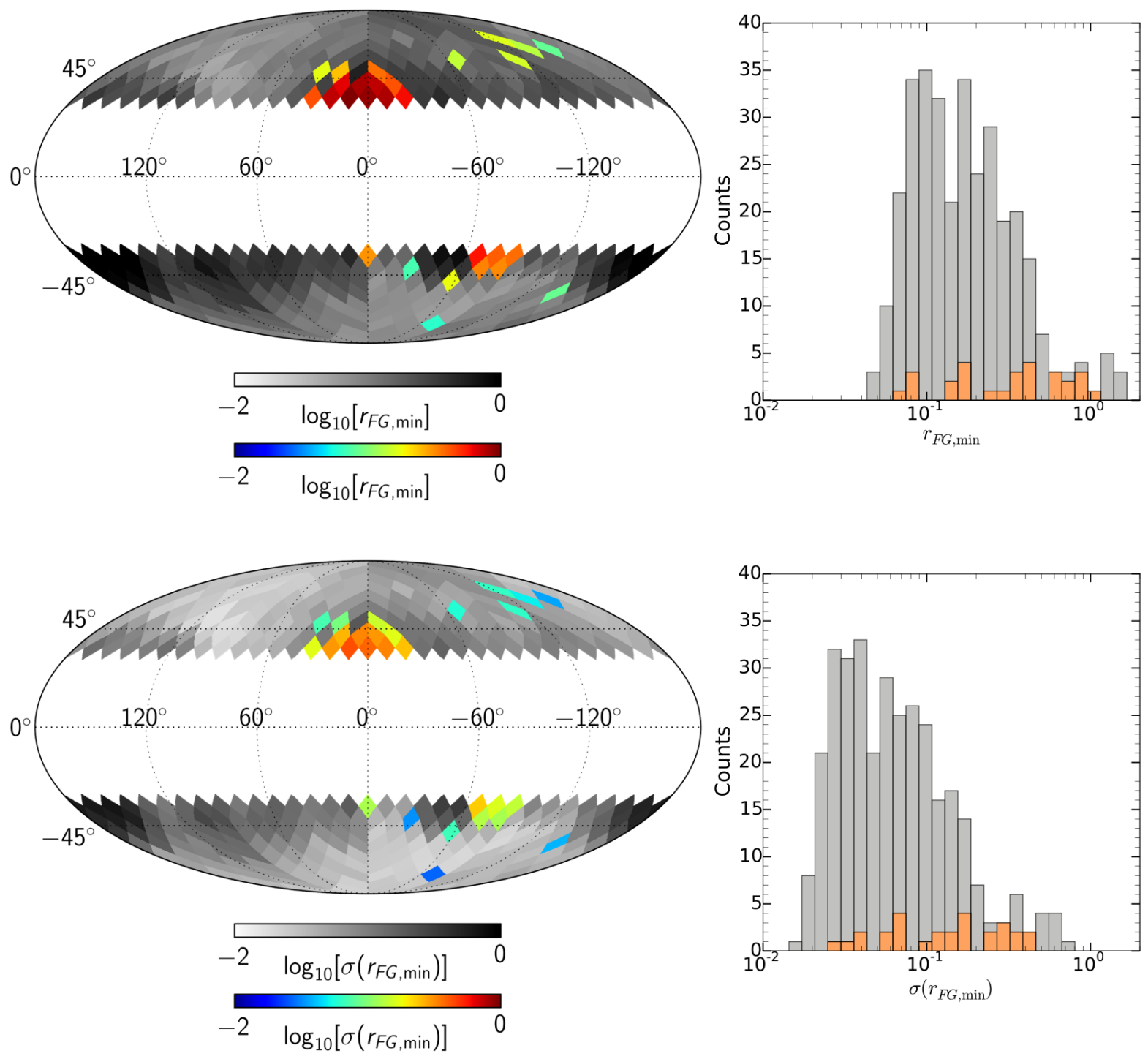

Fig. 4. Minimum estimated foreground emissions $\left(r_{\mathrm{FG}, \min }\right)$ in units of the cosmological tensor-to-scalar ratio (top) and uncertainties on it (bottom). In maps, colored pixels refer to detection, gray pixels to upper limits. Histograms are obtained from the retrieved values reported on maps, orange bars for detection, gray bars for upper limits.

corrections into account to properly include the real frequency response of the instruments (Planck Collaboration IX 2014; Planck Collaboration V 2016).

We recall that synchrotron and dust $B B$ maps of Fig. 3 report the actual value of the spectra in the $\ell_{80}$ bandpower in those pixels corresponding to regions where we have a signal detection above $3 \sigma$, and the upper limit on it in all the other pixels. Nevertheless, we treat detections and upper limits in the same way by rigidly extrapolating the complete maps in frequency. To distinguish among different cases, we divide the sky regions into four classes, depending on whether we reach detections or upper limits for synchrotron and dust, and in the following figures, we use different colors to distinguish them. Classes are defined in Table 2.

Figure 4 shows the minimum foreground amplitude (sum of synchrotron and dust contributions) recovered in each sky patch after frequency extrapolation, together with the associated error. Amplitudes are expressed in terms of $r_{\mathrm{FG}, \min }$, computed by dividing the total $\mathcal{D}_{\ell}^{B B, \mathrm{FG}}$ minimum amplitude of foreground emission in $\mu \mathrm{K}^{2}$ by the value of the CMB primordial $B$ modes power spectrum with tensor-to-scalar ratio $r=1$ in the $\ell_{80}$ bandpower, which is equal to $\sim 6.67 \times 10^{-2} \mu \mathrm{K}^{2}$. Therefore, a value 

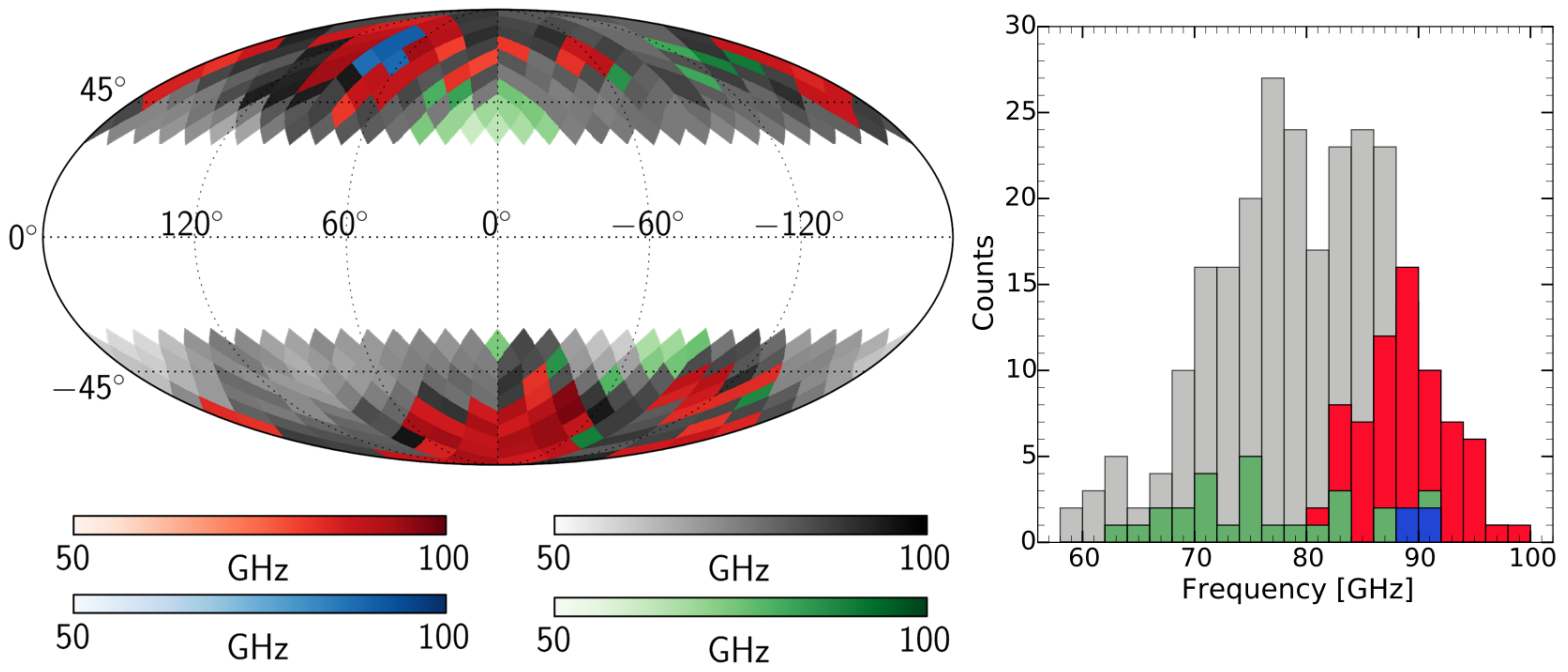

Fig. 5. Map and histogram reporting the frequency at which the minimum of foreground emission is found in each sky region. Color codes as described in Table 2.

of $r_{\mathrm{FG} \text {,min }}=0.1$, for example, means a foreground contribution at the level of a CMB GWs signal with $r=0.1$.

For regions belonging to class (1), maps in Fig. 4 use colored pixels to report the estimated values of $r_{\mathrm{FG} \text {,min }}$ and the associated errors. For each map we also report the histogram of the retrieved values. Uncertainties are calculated as the quadratic sum of the statistical errors coming from the white noise in the sky maps and the errors coming from the uncertainties on the frequency scaling parameters. For regions in classes (2), (3), and (4), we are able to put only upper limits on $r_{\mathrm{FG} \text {,min }}$, which are shown in Fig. 4. We also report the errors on these upper limits, which as before, are the quadratic sum of the statistical errors (included only if we have a detection of at least one of the two kinds of signal) and the frequency scaling ones.

Figure 5 shows the map reporting, for each region, the frequency at which we find the minimum of foreground emission, together with the associated histogram. In this map, we again distinguish the four classes of regions using different color scales. For class (1), since we are dealing with detections, the value of each pixel represents the actual frequency where the minimum of foreground emission is reached. For classes (2) and (3), the reported value represents the frequency at which we set the upper limit on $r_{\mathrm{FG} \text {,min }}$ in the corresponding region. This frequency should be seen as an upper (for class (2)) or a lower limit (for class (3)) on the real frequency at which the foreground emission reaches its actual minimum. For class number (4), each pixel value again represents the frequency that corresponds to the upper limit on $r_{\mathrm{FG}, \min }$, but in this case, we are not able to say whether the real frequency of the foreground minimum lies above or below the reported value.

The recovered amplitudes of $r_{\mathrm{FG} \text {,min }}$, when considering both detections and upper limits, vary between $0.05 \pm 0.02$ and $1.5 \pm 0.7$ (between $0.06 \pm 0.03$ and $1.0 \pm 0.4$ for detections only) in the frequency range 58-98 GHz (with errors between 4 and $7 \mathrm{GHz}$ ). In 123 regions, we find $r_{\mathrm{FG} \text {,min }}<0.12$, the current upper limit on the CMB tensor-to-scalar ratio from $B$-mode observations (BICEP2/Keck and Planck Collaborations 2015).

Table 3 summarizes the results obtained for several regions of interest. The first four regions correspond to those where both synchrotron and dust are detected and $r_{\mathrm{FG} \text {,min }}<0.12$. The remaining ones are the regions where we are able to put the most stringent upper limits on $r_{\mathrm{FG}, \min }$, having $r_{\mathrm{FG}, \min } \lesssim 0.06$. Within the uncertainties of our analysis, we can conclude that there is no region in the sky where the foreground emission demonstrates to contaminate the CMB $B$ modes at levels lower than a signal with tensor-to-scalar ratio $r \sim 0.05$.

As anticipated, we repeated the same analysis considering only the Planck-LFI $30 \mathrm{GHz}$ data as tracer for synchrotron radiation, in order to check whether we obtain results consistent with those retrieved by the correlation $\mathrm{LFI}_{30} \times \mathrm{WMAP}_{K}$. We computed power spectra from the cross-correlation of the two LFI $30 \mathrm{GHz} \mathrm{HM}$ maps. Given the low signal-to-noise ratio of the LFI $30 \mathrm{GHz}$ maps at the considered Galactic latitudes, we only find signal detection at $3 \sigma$ in 5 of the 352 circular regions. Four of these five regions correspond to sky patches where we also find synchrotron detection in the $\mathrm{LFI}_{30} \times \mathrm{WMAP}_{K}$ analysis, and the values of $r_{\mathrm{FG}, \min }$ that we retrieved are compatible at $1 \sigma$. In one region (corresponding to pixel number 62 of the HEALPix $N_{\text {side }}=8$ grid in ring ordering), we find a detection of synchrotron in the $\mathrm{LFI}_{30} \mathrm{HM}_{1} \times \mathrm{HM}_{2}$ case but not in the $\mathrm{LFI}_{30} \times \mathrm{WMAP}_{K}$ case. Here, the value of $r_{\mathrm{FG}}$, min from LFI data is slightly higher than the corresponding upper limit that we are able to put from LFI and WMAP cross-correlation, but still compatible at $1 \sigma$. In all the other regions, where we do not have any detection of synchrotron from LFI data alone, the upper limits we find on $r_{\mathrm{FG}, \min }$ are higher than the constraints that we are able to put then also using WMAP data, as expected given the higher level of noise.

We also evaluated the foreground contamination to $\mathrm{CMB}$ $B$ modes on a larger angular scale, considering the amplitude of thermal dust and synchrotron power spectra in the bandpower including multipoles between 20 and 60, therefore centered at $\ell \simeq 40$. In this estimation we used a second set of 44 larger sky masks, described in Sect. 2, in order tobetter estimate spectral amplitude at low multipoles. The number of regions where we detected both synchrotron and thermal dust is 16 , with recovered value of $r_{\mathrm{FG} \text {, min }}$ ranging between $0.15 \pm 0.06$ and $0.9 \pm 0.3$. In the remaining 28 regions, the upper limits on $r_{\mathrm{FG}, \text { min }}$ vary between $0.08 \pm 0.03$ and $1.1 \pm 0.5$. The frequencies at which we find the minimum of foreground emission, considering both detections and upper limits, range between 59 and $90 \mathrm{GHz}$ with errors between 3 and $6 \mathrm{GHz}$. The analysis on larger scales, 
Table 3. Summary of the characteristics of regions of interests.

\begin{tabular}{|c|c|c|c|c|c|c|}
\hline Pixel number ${ }^{a}$ & $\begin{array}{c}\text { Coordinates }^{b} \\
(b, l)\end{array}$ & $r_{\mathrm{FG}, \min }$ & $\begin{array}{c}r_{\mathrm{FG}, \min } \\
\text { Freq. [GHz] }\end{array}$ & $\begin{array}{c}r_{\mathrm{s}}<0.005 \\
\text { Freq. }[\mathrm{GHz}]\end{array}$ & $\begin{array}{c}r_{\mathrm{d}}<0.005 \\
\text { Freq. [GHz] }\end{array}$ & Class $^{c}$ \\
\hline $\begin{array}{l}51 \ldots \ldots \ldots \\
653 \ldots \ldots \ldots \\
698 \ldots \ldots \ldots \\
753 \ldots \ldots \ldots\end{array}$ & $\begin{array}{c}\left(60.43^{\circ},-153.00^{\circ}\right) \\
\left(-41.81^{\circ},-28.12^{\circ}\right) \\
\left(-54.34^{\circ},-142.50^{\circ}\right) \\
\left(-72.39^{\circ},-75.00^{\circ}\right)\end{array}$ & $\begin{array}{l}0.09 \pm 0.04 \\
0.07 \pm 0.03 \\
0.09 \pm 0.04 \\
0.06 \pm 0.03\end{array}$ & $\begin{array}{l}91 \pm 7 \\
86 \pm 7 \\
88 \pm 7 \\
90 \pm 7\end{array}$ & $\begin{array}{c}135 \pm 9 \\
121 \pm 9 \\
130 \pm 10 \\
126 \pm 8\end{array}$ & $\begin{array}{l}46 \pm 11 \\
45 \pm 10 \\
44 \pm 11 \\
50 \pm 11\end{array}$ & $\begin{array}{l}1 \\
1 \\
1 \\
1\end{array}$ \\
\hline $\begin{array}{l}29 \ldots \ldots \ldots \\
46 \ldots \ldots \\
48 \ldots \ldots \\
67 \ldots \ldots \\
90 \ldots \ldots \\
91 \ldots \ldots \ldots \\
92 \ldots \ldots \ldots \\
93 \ldots \ldots \\
119 \ldots \ldots \\
120 \ldots \ldots \\
152 \ldots \ldots \ldots \\
701 \ldots \ldots \ldots \\
723 \ldots \ldots \\
724 \ldots \ldots \ldots \\
740 \ldots \ldots \\
741 \ldots \ldots \ldots \\
\end{array}$ & $\begin{array}{c}\left(66.44^{\circ}, 123.75^{\circ}\right) \\
\left(60.43^{\circ}, 117.00^{\circ}\right) \\
\left(60.43^{\circ}, 153.00^{\circ}\right) \\
\left(54.34^{\circ}, 112.50^{\circ}\right) \\
\left(48.14^{\circ}, 83.57^{\circ}\right) \\
\left(48.14^{\circ}, 96.43^{\circ}\right) \\
\left(48.14^{\circ}, 109.29^{\circ}\right) \\
\left(48.14^{\circ}, 122.14^{\circ}\right) \\
\left(41.81^{\circ}, 84.37^{\circ}\right) \\
\left(41.81^{\circ}, 95.62^{\circ}\right) \\
\left(35.69^{\circ}, 90.00^{\circ}\right) \\
\left(-54.34^{\circ},-97.50^{\circ}\right) \\
\left(-60.43^{\circ},-81.00^{\circ}\right) \\
\left(-60.45^{\circ},-63.00^{\circ}\right) \\
\left(-66.44^{\circ},-78.75^{\circ}\right) \\
\left(-66.44^{\circ},-56.25^{\circ}\right)\end{array}$ & $\begin{array}{l}0.058 \pm 0.021 \\
0.061 \pm 0.022 \\
0.064 \pm 0.024 \\
0.048 \pm 0.017 \\
0.064 \pm 0.023 \\
0.051 \pm 0.018 \\
0.050 \pm 0.017 \\
0.059 \pm 0.021 \\
0.055 \pm 0.020 \\
0.052 \pm 0.019 \\
0.060 \pm 0.022 \\
0.060 \pm 0.020 \\
0.055 \pm 0.020 \\
0.064 \pm 0.024 \\
0.061 \pm 0.021 \\
0.064 \pm 0.028\end{array}$ & $\begin{array}{l}94 \pm 5 \\
91 \pm 4 \\
90 \pm 5 \\
93 \pm 4 \\
87 \pm 5 \\
94 \pm 5 \\
94 \pm 5 \\
94 \pm 5 \\
87 \pm 4 \\
83 \pm 5 \\
84 \pm 5 \\
86 \pm 4 \\
88 \pm 5 \\
95 \pm 5 \\
88 \pm 5 \\
89 \pm 5\end{array}$ & $\begin{array}{l}129 \pm 4 \\
125 \pm 4 \\
124 \pm 3 \\
122 \pm 4 \\
120 \pm 3 \\
124 \pm 4 \\
125 \pm 4 \\
129 \pm 4 \\
116 \pm 3 \\
109 \pm 3 \\
113 \pm 3 \\
117 \pm 3 \\
118 \pm 3 \\
133 \pm 4 \\
118 \pm 3 \\
121 \pm 3\end{array}$ & $\begin{array}{l}54 \pm 12 \\
51 \pm 11 \\
50 \pm 11 \\
57 \pm 12 \\
48 \pm 11 \\
56 \pm 12 \\
57 \pm 11 \\
54 \pm 11 \\
50 \pm 11 \\
49 \pm 11 \\
47 \pm 10 \\
49 \pm 11 \\
51 \pm 11 \\
53 \pm 12 \\
50 \pm 11 \\
50 \pm 10\end{array}$ & $\begin{array}{l}2 \\
2 \\
2 \\
2 \\
2 \\
2 \\
2 \\
2 \\
2 \\
2 \\
2 \\
4 \\
2 \\
2 \\
2 \\
4\end{array}$ \\
\hline
\end{tabular}

Notes. patches where both synchrotron and thermal dust are detected in the $\ell_{80}$ bandpower and with recovered values for $r_{\mathrm{FG}, \mathrm{min}}<0.12$ (first four rows) and regions where the upper limit on $r_{\mathrm{FG}, \min }$ is below $\sim 0.06$ (remaining sixteen entries). From left to right: HEALPix pixel number, direction of the center, $r_{\mathrm{FG}, \min }$, frequency of minimum emission, frequencies corresponding to synchrotron and dust being negligible within the quoted threshold, region class. ${ }^{(a)}$ Pixel number of the HEALPix $N_{\text {side }}=8$ grid (enumeration starts from zero, ring ordering) associated with the circular region for which spectra are evualated. ${ }^{(b)}$ Galactic coordinates of the region center. ${ }^{(c)}$ Region class as defined in Table 2.

therefore, confirms both the indications from the one at $\ell_{80}$ and the above claim of no evidence for foreground contamination below $r \simeq 0.05$.

Finally, we highlight that, in this analysis, we have made the assumption of having completely uncorrelated synchrotron and thermal dust radiation. Nevertheless, the presence of a correlation would have an impact on the amplitude of the total foreground signal and therefore on the retrieved values of $r_{\mathrm{FG}, \mathrm{min}}$. In our work we evaluate the total foreground $\mathcal{D}_{\ell_{80}}^{B B, F G}$ amplitude as the simple sum of the synchrotron $\left(\mathcal{D}_{\ell=80}^{B B, \mathrm{~s}}\right)$ and dust contribution $\left(\mathcal{D}_{\ell=80}^{B B, \mathrm{~d}}\right)$, including that the cross-correlation leads to

$\mathcal{D}_{\ell \times}^{B B, \mathrm{FG}}=\mathcal{D}_{\ell=80}^{B B, \mathrm{~s}}+\mathcal{D}_{\ell=80}^{B B, \mathrm{~d}}+2 \rho \sqrt{\mathcal{D}_{\ell=80}^{B B, \mathrm{~s}} \mathcal{D}_{\ell=80}^{B B, \mathrm{~d}}}$,

where $\rho$ is the cross-correlation coefficient. Having $\rho \simeq 0.2$, as reported by Choi \& Page (2015), would imply an increase in the value of $r_{\mathrm{FG} \text {,min }}$ of about $20 \%$, while it would not affect the frequency at which the minimum of foreground emission is found.

\subsection{Single foreground contribution}

We can use the same methodology as described above to infer the frequencies at which only one type of foreground significantly contaminates the CMB $B$-mode signal, while the second is below a certain threshold. This information is of particular interest, since it can be used to optimize instrument design, by understanding how many channels are needed and at which frequency, to be able to disentangle the $\mathrm{CMB}$ signal from the Galactic emissions.

We start to estimate the frequency range where the synchrotron emission is negligible. To do this, we extrapolate the
$B B$ synchrotron map in Fig. 3, using the power-law SED described in the previous section We then look in each region for the minimum frequency at which $r_{\mathrm{s}}<0.005$ (with the convention adopted above, $r_{\mathrm{s}}$ is the amplitude of extrapolated synchrotron emission in the $\ell_{80}$ bandpower divided by the amplitude of the CMB $B$ modes with $r=1$ in the same bin). Results are reported in the map and the histogram in the top panel of Fig. 6. We can infer the actual value of this frequency only in the 32 regions where we detect a synchrotron $B$-mode signal (green pixels in map). For all the other sky patches, we put upper limits. Considering both detections and upper limits, the retrieved frequencies vary between $\sim 110$ and $\sim 160 \mathrm{GHz}$ across the sky (with errors between $\sim 3$ and $\sim 10 \mathrm{GHz}$ ). From this analysis, we can therefore conclude that, given the current knowledge we have on polarized synchrotron radiation, it is not possible to exclude that CMB $B$-mode measurements at frequencies below $\sim 110 \mathrm{GHz}$ are contaminated by this kind of Galactic emission at the level of $r=0.005$, in any region of the sky. Considering a most stringent threshold on the amplitude of synchrotron with $r_{\mathrm{s}}<0.001$, this frequency increases at $\sim 150 \mathrm{GHz}$.

Similarly we rescale the $B B$ thermal dust map in Fig. 3 to find the maximum frequency at which $r_{\mathrm{d}}<0.005$ for each region. Results are shown in the bottom panel of Fig. 3, for the regions where we have a detection of the thermal dust signal (green pixels) and for those where we can only put lower limits on the value of this frequency (blue pixels). Considering all sky regions, the maximum value we find is $57 \pm 12 \mathrm{GHz}$, dropping to about $40 \mathrm{GHz}$ if we impose $r_{\mathrm{d}}<0.001$.

Table 3 reports, for the regions of interest, the retrieved minimum and maximum frequencies at which $r_{\mathrm{s}}<0.005$ and $r_{\mathrm{d}}<0.005$, respectively. 

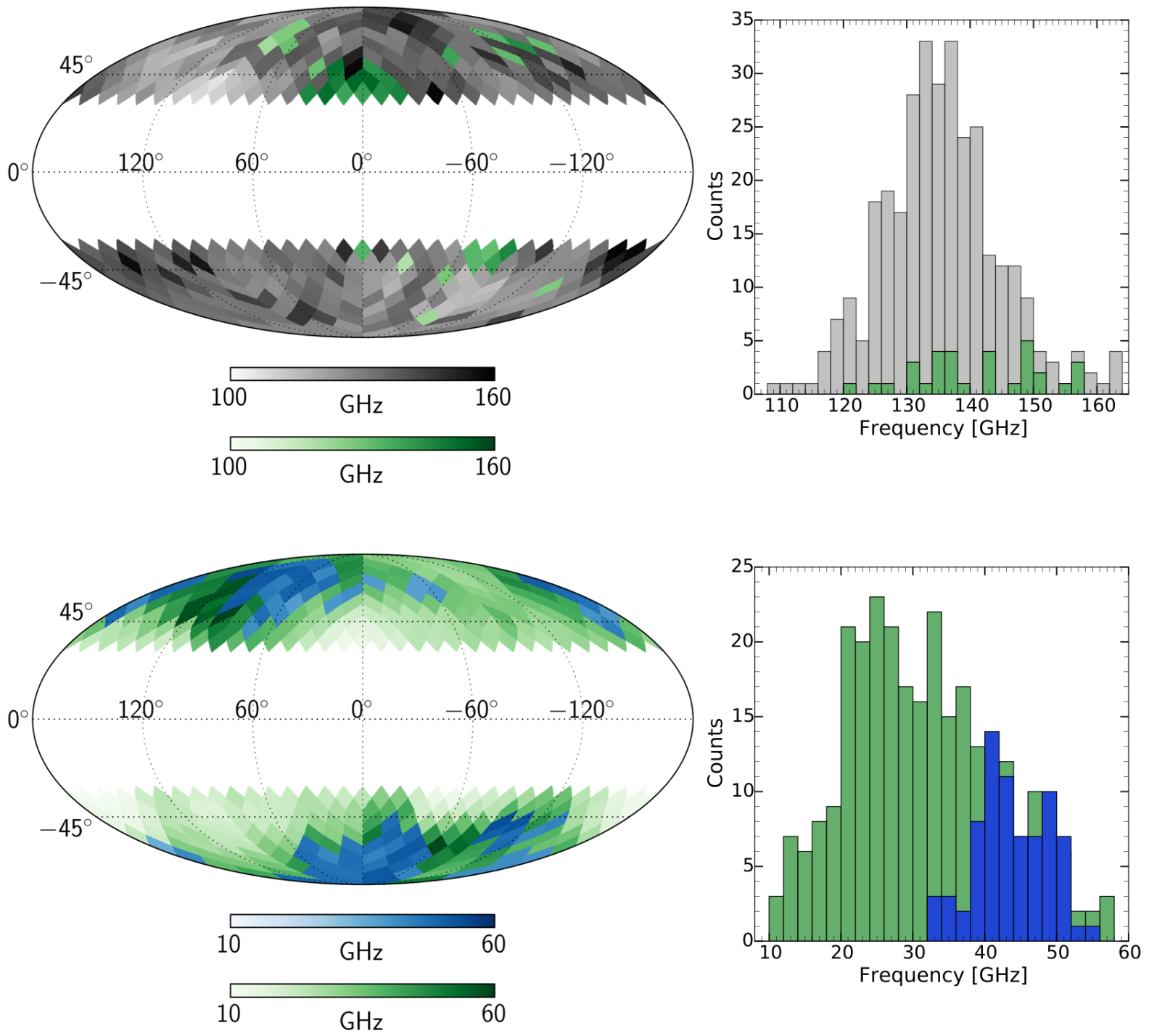

Fig. 6. Frequency at which synchrotron and thermal dust contamination to CMB B modes drops below $r=0.005$ (top and bottom panels, respectively). Green pixels for detections, gray pixels for upper limits, blue pixels for lower limits.

\section{Discussion and conclusions}

We quantify the contamination from polarized diffuse Galactic synchrotron and thermal dust emissions to the $B$ modes of CMB anisotropies on degree angular scales. Our analysis is based on the data made available from the Planck and WMAP satellites at Galactic latitudes with $|b|>20^{\circ}$, and exploit the Xpure power spectrum estimator, which we validate on suitable simulations. We measure the synchrotron emission evaluating cross-spectra of the $30 \mathrm{GHz}$ Planck-LFI channel with the WMAP- $K$-band, centered at $22 \mathrm{GHz}$. The thermal dust emission is quantified using the $353 \mathrm{GHz}$ channel of the HFI. We assign uncertainties to power spectra using the noise covariance matrices of the adopted maps.

We focus on the $\ell_{80}$ bandpower in the angular domain, corresponding to the angular scales where the CMB $B$-modes recombination bump peaks and where, currently, suborbitals experiments are focusing to constrain $r$. In particular we evaluate power spectra of foreground emissions in 352 small circular regions of the sky with $f_{\text {sky }} \simeq 1.5 \%$. In 28 sky regions, both synchrotron and dust spectra are measurable, and there, extrapolation in frequency suggests a minimum foreground level which is equivalent to a tensor-to-scalar ratio $r_{\mathrm{FG} \text {,min }}$ between $\sim 0.06$ and $\sim 1.0$, in the frequency interval between about 60 and $100 \mathrm{GHz}$. In all the regions where synchrotron or dust $B$-mode polarized signal is not detectable at $3 \sigma$ significance, we put upper limits on the minimum foreground contamination, finding values of $r_{\mathrm{FG}, \text { min }}$ between $\sim 0.05$ and $\sim 1.5$ in the frequency range 60-90 GHz.

Table 3 summarized the obtained results in twenty regions:

- the four regions where both thermal dust and synchrotron are detected in the $\ell_{80}$ bin and with a recovered value $r_{\mathrm{FG} \text {,min }}<$ 0.12 ;

- the sixteen regions where the upper limit on $r_{\mathrm{FG}, \min }$ is $\lesssim 0.06$.

We also estimate the frequency range where the only significant contamination arises from a single foreground component. On the high frequency side, where the dust emission dominates, the synchrotron is estimated to be negligible (with $r_{\mathrm{s}}<0.005$ ) only at frequencies higher than $160 \mathrm{GHz}$ (considering all the regions analyzed). The upper limits we put on this frequency are never 


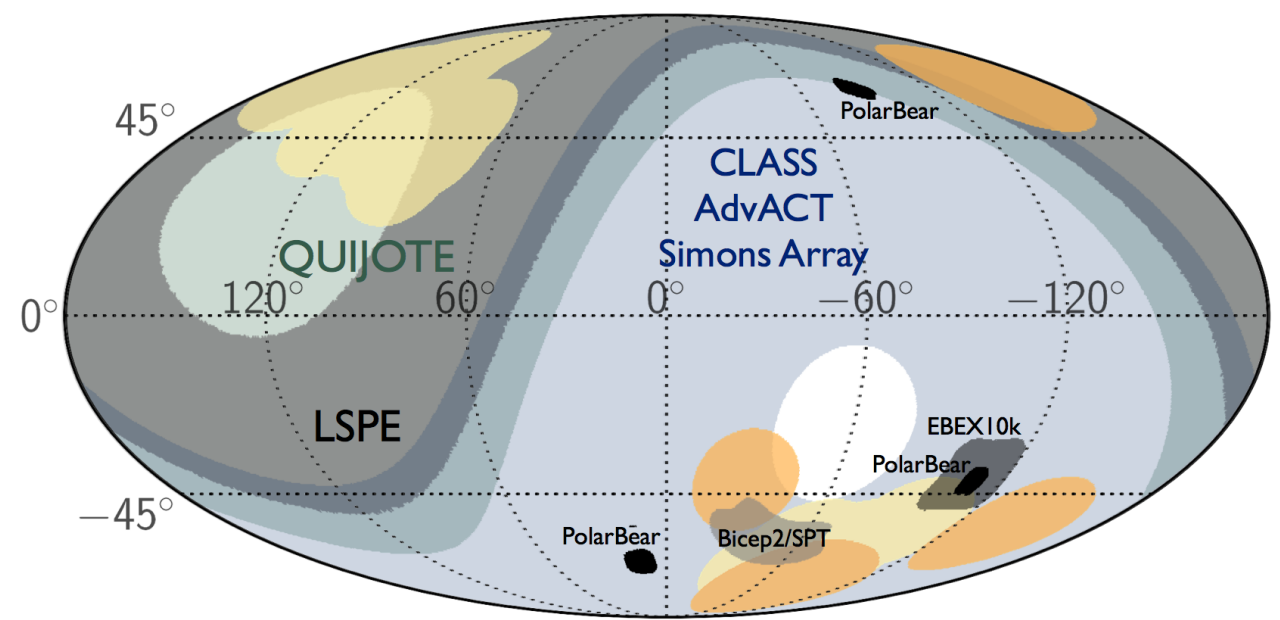

Fig. 7. Observed or targeted sky patches for the quoted experiments versus locations of the regions described in Table 3. Dark yellow regions represent the first four entries of Table 3, light yellow color refers to the remaining sixteen.

less than $\sim 110 \mathrm{GHz}$. On the low-frequency side, the dust drops at the same level in the $10-60 \mathrm{GHz}$ frequency range.

The same twenty sky patches reported in Table 3 are also displayed in the map in Fig. 7, together with several regions that have been, or will be, observed by current and future ground-based or ballon-borne CMB experiments. In particular we consider the cases of the LSPE (LSPE Collaboration 2012) and EBEX10K (Chapman et al. 2014) proposed balloons, the CLASS (Essinger-Hileman et al. 2014), advACT (Calabrese et al. 2014), QUIJOTE (Génova-Santos et al. 2015), Simons Array ground-based experiment (Suzuki et al. 2016), as well as the areas observed by BICEP2/Keck Array (BICEP2 Collaboration I 2014; BICEP2 and Keck Array Collaborations 2015), SPT (Benson et al. 2014) and PolarBear (The Polarbear Collaboration 2014).

In the southern Galactic hemisphere we find a large region of the sky characterized by low upper limits on $r_{\mathrm{FG}, \mathrm{min}}$. This confirms that this area appears to be among the cleanest in the sky. However, in the proximity of this region (in particular at the edges of the area observed by BICEP2) we have a few patches where synchrotron $B$ modes are detected with an extrapolated amplitude corresponding to $r_{\mathrm{s}} \approx 0.03$ at about $90 \mathrm{GHz}$. This is consistent with the recent analysis of Keck-BICEP2 collaborations (BICEP2 and Keck Array Collaborations 2016) that improves the current upper limits on tensor-to-scalar ratio to $r<0.09$, highlighting a small synchrotron contribution in this field (the first panel in Fig. 3 of BICEP2 and Keck Array Collaborations 2016, in particular, shows a small excess in the cross-correlation between WMAP $K$-band data with Keck and BICEP2 measurements). Moreover, close to this area of the sky, we detect synchrotron $E$ modes (see Fig. 3), which can trace possible $B$ modes at a level below the sensitivity of our analysis. In summary, our analysis confirms that this region in the southern Galactic hemisphere contains little contribution from polarized synchrotron emissions compared to other regions in the sky. The presence of these low residuals, however, should not be neglected in view of experimental efforts aimed at detecting $r$ at levels $r<0.03$ at frequencies below $\sim 100 \mathrm{GHz}$. Similarly, there is a large area with low upper limits in the north Galactic hemisphere, where we do not detect synchrotron $B$ modes signal, but we observe synchrotron $E$ modes.

Several experiments are currently observing, or will observe in the near future, large portions of the sky, with both low- and high-frequency channels (see, for example in Fig. 7, the regions covered by CLASS, AdvACT, Simons Array, QUJIOTE, and LSPE), covering also the regions of interested highlighted in this work, giving the possibility to better constrain both synchrotron and thermal dust emissions.

In conclusion, our results indicate that, with the current sensitivity at low frequency, it is not possible to exclude the presence of synchrotron contamination to CMB cosmological $B$ modes measurements at frequency $\lesssim 100 \mathrm{GHz}$ anywhere. At these frequencies, more accurate data are essential for understanding the synchrotron polarized component, and eventually remove its contamination to $\mathrm{CMB}$ measurements through foreground cleaning. Restricting the observations to higher frequencies $(\gtrsim 110 \mathrm{GHz}$ ) and focusing only on dust contamination would be an option for targeting a CMB $B$ mode signal with $r \simeq 0.01$, since in several sky regions, the synchrotron emission drops at $r_{\mathrm{s}}<0.005$ at these frequencies. Nevertheless, the observation of a more fainter GWs signal (with $r<0.01$ ) will require instruments with both low- and high-frequency channels to monitor, with high accuracy, both kinds of foreground emission.

Acknowledgements. We gratefully acknowledge support from ASI/INAF Agreement 2014-024-R.0 for the Planck LFI Activity of Phase E2. N.K. acknowledges Maurizio Tomasi for careful reading the manuscript. C.B. acknowledges partial support by the INDARK INFN Grant.

\section{References}

Baccigalupi, C. 2003, New Astron. Rev., 47, 1127

Bennett, C. L., Larson, D., Weiland, J. L., et al. 2013, ApJS, 208, 20

Benson, B. A., Ade, P. A. R., Ahmed, Z., et al. 2014, in SPIE Conf. Ser., 9153, 91531

BICEP2 Collaboration I. 2014, Phys. Rev. Lett., 112, 241101

BICEP2 and Keck Array Collaborations 2015, ApJ, 811, 126

BICEP2/Keck and Planck Collaborations 2015, Phys. Rev. Lett., 114, 101301

BICEP2 and Keck Array Collaborations. 2016, Phys. Rev. Lett., 116, 031302

Calabrese, E., Hložek, R., Battaglia, N., et al. 2014, J. Cosmology Astropart. Phys., 8, 010

Chapman, D., Aboobaker, A. M., Ade, P., et al. 2014, in AAS Meeting Abstracts, 223, 407.03

Choi, S. K., \& Page, L. A. 2015, JCAP, 12, 020

Essinger-Hileman, T., Ali, A., Amiri, M., et al. 2014, in SPIE Conf. Ser., 9153 , 1

Fraisse, A. A., Ade, P. A. R., Amiri, M., et al. 2013, J. Cosmol. Astropart. Phys., 4,47

Fuskeland, U., Wehus, I. K., Eriksen, H. K., \& Næss, S. K. 2014, ApJ, 790, 104 
N. Krachmalnicoff et al.: Characterization of foreground emission for CMB $B$-mode observations

Génova-Santos, R., Rubiño-Martín, J. A., Rebolo, R., et al. 2015, MNRAS, 452, 4169

Gold, B., Odegard, N., Weiland, J. L., et al. 2011, ApJS, 192, 15

Górski, K. M., Hivon, E., Banday, A. J., et al. 2005, ApJ, 622, 759

Grain, J., Tristram, M., \& Stompor, R. 2009, Phys. Rev. D, 79, 123515

Kogut, A., Dunkley, J., Bennett, C. L., et al. 2007, ApJ, 665, 355

LSPE Collaboration. 2012, in SPIE Conf. Ser., 8446, 7

Page, L., Hinshaw, G., Komatsu, E., et al. 2007, ApJS, 170, 335

Planck Collaboration IX. 2014, A\&A, 571, A9

Planck Collaboration I. 2015, A\&A, submitted [arXiv: 1502 .01582]

Planck Collaboration II. 2016, A\&A, in press,

DOI: $10.1051 / 0004-6361 / 201525818$

Planck Collaboration V. 2016, A\&A, in press,

DOI: $10.1051 / 0004-6361 / 201526632$

Planck Collaboration VI. 2016, A\&A, in press, DOI: $10.1051 / 0004-6361 / 201525813$

Planck Collaboration VII. 2016, A\&A, in press, DOI: $10.1051 / 0004-6361 / 201525844$
Planck Collaboration VIII. 2016, A\&A, in press,

DOI: $10.1051 / 0004-6361 / 201525820$

Planck Collaboration X. 2015, A\&A, submitted [arXiv: 1502 .01588]

Planck Collaboration XIII. 2015, A\&A, submitted [arXiv: 1502 . 01589]

Planck Collaboration XX. 2015, A\&A, submitted [arXiv: 1502.02114]

Planck Collaboration XXV. 2015, A\&A, submitted [arXiv: 1506. 06660]

Planck Collaboration XXVI. 2016, A\&A, in press,

DOI: 10.1051/0004-6361/201526914

Planck Collaboration Int. XIX. 2015, A\&A, 576, A104

Planck Collaboration Int. XXII. 2015, A\&A, 576, A107

Planck Collaboration Int. XXX. 2016, A\&A, 586, A133

QUBIC Collaboration 2011, Astropart. Phys., 34, 705

Smith, K. M. 2006, Phys. Rev. D, 74, 083002

Suzuki, A., Ade, P., Akiba, Y., et al. 2016, J. Low Temp. Phys., in press, DOI: $10.1007 / \mathrm{s} 10909-015-1425-4$

The Polarbear Collaboration. 2014, ApJ, 794, 171

Tristram, M., Macías-Pérez, J. F., Renault, C., \& Santos, D. 2005, MNRAS, 358, 833 